\title{
Retinal Targets ALDH Positive Cancer Stem Cell and Alters the Phenotype of Highly Metastatic Osteosarcoma Cells
}

\author{
Xiaodong Mu, ${ }^{1}$ Stuti Patel, ${ }^{2}$ Damel Mektepbayeva, ${ }^{3}$ Adel Mahjoub, \\ Johnny Huard, ${ }^{1}$ and Kurt Weiss ${ }^{2}$ \\ ${ }^{1}$ Department of Orthopaedic Surgery, University of Texas Health Science Center at Houston, Houston, TX 77030, USA \\ ${ }^{2}$ Cancer Stem Cell Laboratory, Department of Orthopaedic Surgery, University of Pittsburgh, Pittsburgh, PA 15219, Kazakhstan \\ ${ }^{3}$ Laboratory of Cellular Technology, Nazarbayev University, Astana, Akmola 010000, USA \\ Correspondence should be addressed to Kurt Weiss; weiskr@upmc.edu
}

Received 12 October 2015; Accepted 17 November 2015

Academic Editor: Kanya Honoki

Copyright (C) 2015 Xiaodong Mu et al. This is an open access article distributed under the Creative Commons Attribution License, which permits unrestricted use, distribution, and reproduction in any medium, provided the original work is properly cited.

\begin{abstract}
Aldehyde dehydrogenase (ALDH) is a cancer stem cell marker. Retinoic acid has antitumor properties, including the induction of apoptosis and inhibition of proliferation. Retinal, the precursor of retinoic acid, can be oxidized to retinoic acid by dehydrogenases, including ALDH. We hypothesized that retinal could potentially be transformed to retinoic acid with higher efficiency by cancer stem cells, due to the higher ALDH activity. We previously observed that ALDH activity is greater in highly metastatic K7M2 osteosarcoma (OS) cells than in nonmetastatic K12 OS cells. We also demonstrated that ALDH activity correlates with clinical metastases in bone sarcoma patients, suggesting that ALDH may be a therapeutic target specific to cells with high metastatic potential. Our current results demonstrated that retinal preferentially affected the phenotypes of ALDH-high K7M2 cells in contrast to ALDH-low K12 cells, which could be mediated by the more efficient transformation of retinal to retinoic acid by ALDH in K7M2 cells. Retinal treatment of highly metastatic K7M2 cells decreased their proliferation, invasion capacity, and resistance to oxidative stress. Retinal altered the expression of metastasis-related genes. These observations indicate that retinal may be used to specifically target metastatic cancer stem cells in OS.
\end{abstract}

\section{Introduction}

Retinoids are a class of compounds comprised of several signaling molecules, such as retinoic acid and retinaldehyde, that are structurally related to vitamin A (retinol) [1]. These molecules play a crucial role in the regulation of various cellular processes. Retinoids have been shown to exert a tumorsuppressive effect on cells based on their interaction with cyclins and cyclin-dependent kinases (CDKs) to prevent cellcycle progression [1]. Additionally, they also influence cancer cell differentiation and apoptosis [2]. Many cancers have been shown to have abnormally low levels of various retinoids.

Retinoic acid, a derivative of retinol, has been shown to inhibit proliferation of malignant tumors and induce the apoptosis and differentiation in various types of cancer cells $[1,3-6]$. Due to retinoic acid's antitumor properties, its role is being investigated in the treatment of various malignancies [3]. It is currently used in the treatment of acute promyelocytic leukemia and has been shown to result in complete remission [7]. Retinoic acid is derived from its precursor, all-trans-retinal (also called retinaldehyde or vitamin A aldehyde), by the action of dehydrogenases, including aldehyde dehydrogenase (ALDH) [8]. Previous studies have demonstrated that high ALDH activity predicted poor survival in various types of cancers $[9,10]$, such as breast cancer [11], epithelial cancer [12], and sarcoma [13]. ALDH activity is often specifically upregulated in cancer stem cells and has been recognized as a marker for cancer stem cells $[10,14-$ 16]. Therefore, we believe it is reasonable to hypothesize that, because of the higher ALDH activity in cancer stem cells, retinal could be more efficiently transformed to retinoic acid by cancer stem cells, and thus preferentially induce the apoptosis and alteration of these cells. 
Osteosarcoma (OS) is the most common primary tumor of the bone. It has a bimodal age distribution with a major peak during the second decade of life and a second smaller peak observed in patients over 50 years old. The lung is the most common site of metastatic spread, and survival is ultimately determined by the presence of absence of pulmonary metastatic disease. Deaths related to OS are thus the result of metastasis to the lung rather than the primary tumor itself. The prognosis of patients with OS has not improved in the past several decades due to the lack of treatment specifically targeting OS cell metastatic potential. Due to our limited understanding of the biology of sarcoma metastasis, this problem remains unsolved.

We have demonstrated the role of various cytokines and signaling pathways in OS metastasis using two related cell populations of a spontaneously occurring murine OS [1720]. These cell lines, $\mathrm{K} 12$ and K7M2, differ in their metastatic potential, with $\mathrm{K} 7 \mathrm{M} 2$ showing more features of cancer stem cells and a much greater metastatic rate to the lungs compared to K12 cells. The difference in their characteristics and metastatic potentials demonstrated that K7M2 and K12 feature different activation statuses of multiple biologic factors and signaling pathways: ALDH, Notch1, andmammalian target of rapamycin (mTOR). The inhibition of these signaling pathways alters OS cell behavior in vitro [17-20]. We observed that K7M2 cells demonstrated greater ALDH gene expression and activity than the less metastatic K12 cells. Additionally, ALDH activity was found to be greater in cultured cells from bone sarcoma patients who experienced clinical metastasis [21]. We thus hypothesized that ALDH might represent a means to specifically target sarcoma cells with high metastatic potential.

Our current research investigates the effect of novel treatment approaches to these metastasis-related genes. In the present study, we sought to take advantage of the increased level of ALDH activity in highly metastatic K7M2 OS cells to study the effects of retinal on cell growth and metastasis. Given the ability of retinal to be transformed into retinoic acid by ALDH and the high level of expression of ALDH in cancer stem cells, we hypothesized that treatment with retinal would selectively induce apoptosis and suppress tumor growth in K7M2 OS cells.

\section{Materials and Methods}

2.1. Cell Culture and Retinal Treatment. K12 and K7M2 cells were cultured in proliferation medium (10\% FBS in DMEM). 5,000 cells were seeded in each well of a 12 -well plastic plate. Cells were treated with either $1 \mu \mathrm{g} / \mathrm{mL}$ or $5 \mu \mathrm{g} / \mathrm{mL}$ of all-transretinal (Sigma) dissolved in $1 \mathrm{~mL}$ of proliferation medium. K7M 2 cells treated with $1 \mu \mathrm{L}$ of DMSO served as control. Cells were incubated for 2 days, before being fixed for observation or harvested for mRNA isolation.

2.2. Fluorescence-Activated Cell Sorting (FACS) Analysis of ALDH Activity and Sorting of Cells. ALDH enzymatic activity was determined using the Aldefluor Kit (STEMCELL Technologies). Cultured K7M2 cells from treatment as well as control groups were resuspended in Aldefluor buffer
TABle 1: Primer sequences.

\begin{tabular}{ll}
\hline Gene & Primer sequence \\
\hline \multirow{2}{*}{ GAPDH } & $\begin{array}{l}\text { Forward: TCCATGACAACTTTGGCATTG } \\
\text { Reverse: TCACGCCACAGCTTTCCA }\end{array}$ \\
\hline \multirow{2}{*}{ Notch1 } & $\begin{array}{l}\text { Forward: GCCGCAAGAGGCTTGAGAT } \\
\text { Reverse: GGAGTCCTGGCATCGTTGG }\end{array}$ \\
\hline \multirow{2}{*}{ Hes1 } & $\begin{array}{l}\text { Forward: CCAGCCAGTGTCAACACGA } \\
\text { Reverse: AATGCCGGGAGCTATCTTTCT }\end{array}$ \\
\hline \multirow{2}{*}{ BMP2 } & $\begin{array}{l}\text { Forward: TCTTCCGGGAACAGATACAGG } \\
\text { Reverse: TGGTGTCCAATAGTCTGGTCA }\end{array}$ \\
\hline \multirow{2}{*}{ Fkt } & $\begin{array}{l}\text { Forward: ATGAACGACGTAGCCATTGTG } \\
\text { Reverse: TTGTAGCCAATAAAGGTGCCAT }\end{array}$ \\
\hline Forward: \\
& $\begin{array}{l}\text { TGACCTAACTCGAGGAGGAGCTGGAATC } \\
\text { AAGTTTGAGGCAGTTAAAATTATGGCTGAAGC }\end{array}$ \\
\hline ALDH1 & $\begin{array}{l}\text { Forward: GACAGGCTTTCCAGATTGGCTC } \\
\text { Reverse: AAGACTTTCCCACCATTGAGTGC }\end{array}$ \\
\hline Klotho & $\begin{array}{l}\text { Forward: CCCAAACCATCTATGAAAC } \\
\text { Reverse: CTACCGTATTCTATGCCTTC }\end{array}$ \\
\hline
\end{tabular}

$\left(1 \times 10^{6}\right.$ cells $\left./ \mathrm{mL}\right)$ and incubated at $37^{\circ} \mathrm{C}$ as per manufacturer's instructions. During the cell sorting process, cells were washed in Aldefluor buffer and maintained at $4^{\circ} \mathrm{C}$. FL1 channel of a BD FACSAria Cell Sorting System and FACSDiva software (version 6.1.2; Becton, Dickinson and Company, San Jose, CA) was used to assess ALDH activity. Fluorescenceactivated cell sorting (FACS) was utilized to collect cells according to their fluorescence intensity, which corresponds to cells' ALDH activity level. Cells with high and low ALDH levels were separately isolated and cultured.

2.3. Semiquantitative Reverse Transcription Polymerase Chain Reaction (RT-PCR). RNeasy plus mini kit (Qiagen) and the iScript cDNA Synthesis kit (Bio-Rad) were used to extract RNA from the cells and generate cDNA, respectively. RT-PCR was performed using a Bio-Rad MyiQ thermal cycler (BioRad). Table 1 shows the sense and antisense primers for RTPCR and their product. Following cycling parameters were used for all reactions: $94^{\circ} \mathrm{C}$ for 5 minutes; 30 cycles of the following: denature for 45 seconds at $95^{\circ} \mathrm{C}$, anneal for 30 seconds $\left(53^{\circ} \mathrm{C}-56^{\circ} \mathrm{C}\right)$, and extend for 45 seconds at $72^{\circ} \mathrm{C}$. Expression of target genes was normalized to the expression of GAPDH, which served as a control gene. ImageJ software (version $1.32 \mathrm{j}$, National Institutes of Health, Bethesda, MD) was used to perform RT-PCR analysis. The integrated density of bands was calculated based on product of the area and the mean gray value. All molecular bands were normalized to GAPDH.

2.4. Cell In Vitro Invasion Assay. In vitro invasion capacity of K7M2 cells treated with retinal was compared to control cells using a real-time cell invasion and migration (RT-CIM) assay system (ACEA Biosciences, Inc.), with a 16-well transwell plate (CIM-plate 16, Roche Diagnostics GmbH). The surface 
of the wells in the upper chamber was coated with Matrigel (BD BioSciences, Bedford, MA, USA) of different concentrations $(2.5 \%, 5 \%$, and $10 \%)$. Serum-containing medium $(10 \%$ FBS) was added to the wells of the lower chamber. Cells $(4 \times$ $10^{4}$ per well) in serum-free medium were seeded in the upper chamber. The migration of the cells through the Matrigel was monitored by the system every 15 minutes for 24 hours. Data analysis was carried out using RTCA Software 1.2 supplied with the instrument.

2.5. Actin Staining. Cells from each group were treated with phalloidin conjugated with Alexa Fluor 488 (Invitrogen) to observe actin organization. Treatment and control cells were grown overnight in proliferation medium in 12 -well plates (50,000 cells/plate). The following day, the plates were washed twice with PBS, fixed in $3.7 \%$ formaldehyde solution for 10 minutes at room temperature, and washed two more times with PBS. The cells were then permeabilized in $0.1 \%$ Trition X-100 for 20 minutes and washed again with PBS. $5 \mu \mathrm{L}$ of methanolic stock solution phalloidin, $200 \mu \mathrm{L}$ PBS, and $1 \%$ BSA were added to each well and allowed to stand for $20 \mathrm{~min}$ utes, before being washed again with PBS. $300 \mu \mathrm{L}$ of $300 \mathrm{nM}$ DAPI was used for nuclear staining.

2.6. Statistical Analysis. Statistical analysis of all experiments for this study was carried out using data from at least three samples from each treatment group. Statistical significance was evaluated using Student's $t$-test. Difference between groups was considered significant for $p$ value $<0.05$.

\section{Results}

3.1. Retinal Treatment of Highly Metastatic K7M2 Cells Decreased the Proliferation Capacity and Resistance to Oxidative Stress. K7M2 cells have been shown to feature much higher ALDH activity compared with K12 cells [17, 18]. K7M2 cells treated with all-trans-retinal $(5 \mu \mathrm{g} / \mathrm{mL})$ for 2 days were observed to have fewer cells on the culture dishes compared with the nontreated K7M2 cells (Figure 1(a)). Actually, compared to retinal-treated $\mathrm{K} 12$ cells, the proliferation of retinaltreated K7M2 cells was much more obviously decreased (Figure 1), indicating preferentially increased apoptosis in retinaltreated K7M2 cells. Furthermore, retinal-treated K7M2 cells also displayed higher levels of apoptosis induced by exposure to hydrogen peroxide $(250 \mu \mathrm{M}, 1 \mathrm{hr})$, as seen by propidium iodide (PI) staining (data not shown). Our previous results have shown that the sorted ALDH-high K7M2 cells are more resistant to oxidative stress-induced cell death, compared with the sorted ALDH-low cells [17]. Treatment of ALDHhigh K7M2 cells with retinal $(5 \mu \mathrm{g} / \mathrm{mL})$ for 2 days significantly increased the ratio of apoptotic cells induced by exposure to hydrogen peroxide, compared to nontreated control ALDHhigh K7M2 cells (Figure 2). The apoptotic effect was much more pronounced than in ALDH-low K7M2 cells. This observation indicates that the resistance to oxidative stress of K7M2 cells was preferentially decreased by retinal in cells with high ALDH activity.
3.2. Retinal Treatment of K7M2 Cells Resulted in Modified Gene Expression of Metastasis-Related Factors and Reduced ALDH Activity. Semiquantitative PCR analysis of K7M2 cells with and without retinal treatment was carried out to compare the expression levels of various signaling molecules involved in cell proliferation, apoptosis, and metastasis. Treatment with retinal resulted in the downregulation of Notch signaling as evidenced by decreased expression of Notchl and Hes1. Further, there was also a decrease in bone morphogenetic protein 2 (BMP2), cMyc, and ALDH levels while Klotho, a tumor suppressor, was up-egulated with retinal treatment (Figure 3(a)). Additionally, ALDH activity assay of K7M2 cells verified that retinal treatment (both $1 \mu \mathrm{g}$ / $\mathrm{mL}$ and $5 \mu \mathrm{g} / \mathrm{mL}$ ) was able to reduce the ALDH activity of K7M2 cells (Figure 3(b)).

3.3. Retinal Treatment of K7M2 Cells Altered Cell Morphological Characteristics and Decreased Invasion Capacity. Our previous results demonstrated that K7M2 cells feature different cell morphology than K12 cells and have significantly higher migration and invasion capacities in vitro $[17,18]$. Here, actin staining by phalloidin demonstrated that K7M2 cells treated with retinal had undergone obvious alterations in migrationrelated cell morphological characteristics with reduced appearance of invadopodia, which play critical roles in cell migration/invasion (Figure 4(a)). Finally, in vitro invasion assays further displayed that treatment of K7M2 cells with retinal $(5 \mu \mathrm{g} / \mathrm{mL}, 2$ days) resulted in decreased cell invasion compared with untreated cells (Figure 4(b)).

\section{Discussion}

All-trans-retinal is a precursor of retinoic acid and can be transformed into retinoic acid by dehydrogenases, including ALDH. Retinoic acid has been shown to induce differentiation of hematopoietic and neural stem cells. Retinoic acid appears to induce apoptosis and differentiation in a number of cancers and has been successfully used to treat acute promyelocytic leukemia $[1,7]$. However, the role of retinal as an antiproliferative agent in OS has not been studied. Based on the fact that retinal can be transformed to retinoic acid by ALDH, and ALDH activity is typically higher in cancer stem cells $[9,15,18]$, we investigated the effect that retinal would have on highly metastatic OS cells.

Our study revealed that retinal was able to preferentially target the highly metastatic K7M2 cells which feature greater ALDH activity and other stem cell characteristics, by repressing their proliferation and inducing the apoptosis of these cells. The role of retinal in decreasing cell proliferation and cell survival was demonstrated by exposing cells to oxidative stress using hydrogen peroxide. ALDH-high K7M2 cells exhibited a greater increase in apoptosis compared to ALDH-low K7M2 cells. Additionally, our RT-PCR results also showed that retinal treatment results in the downregulation of various genes involved in cell-proliferation and cell survival. Namely, the expression level of Notch1, Hesl, cMyc, and BMP2 was decreased in a dose-dependent manner. Further, there was an upregulation of Klotho, a tumor suppressor gene. 
K7M2-control

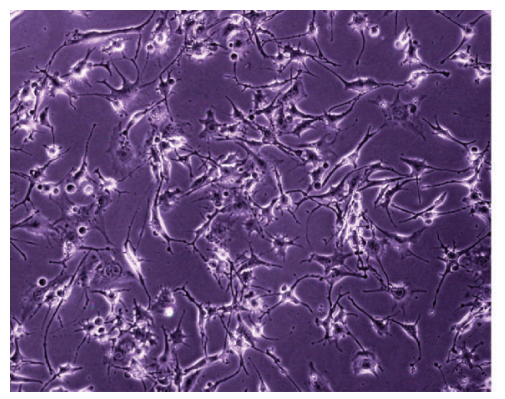

K7M2-retinal $(5 \mu \mathrm{g} / \mathrm{mL})$

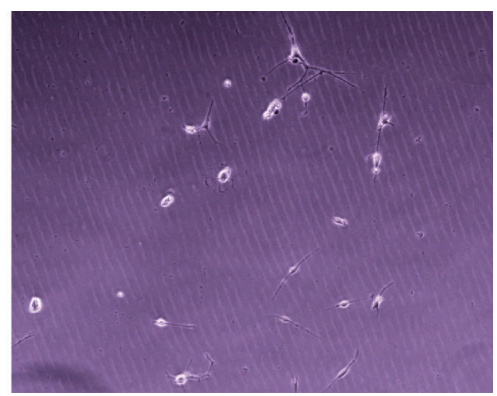

K7M2 cells

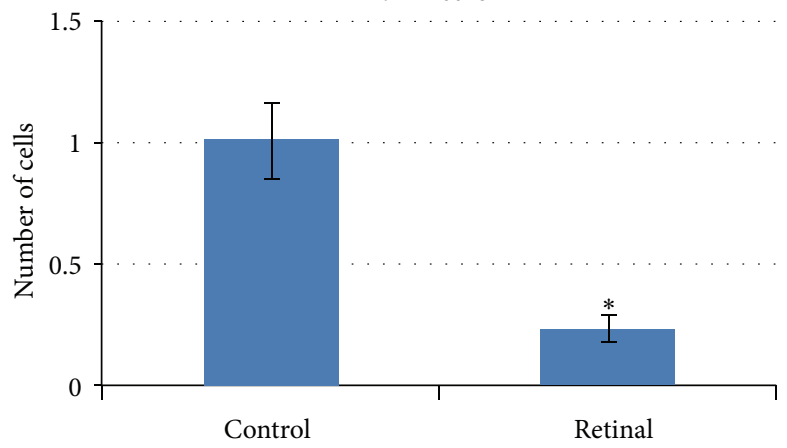

(a)
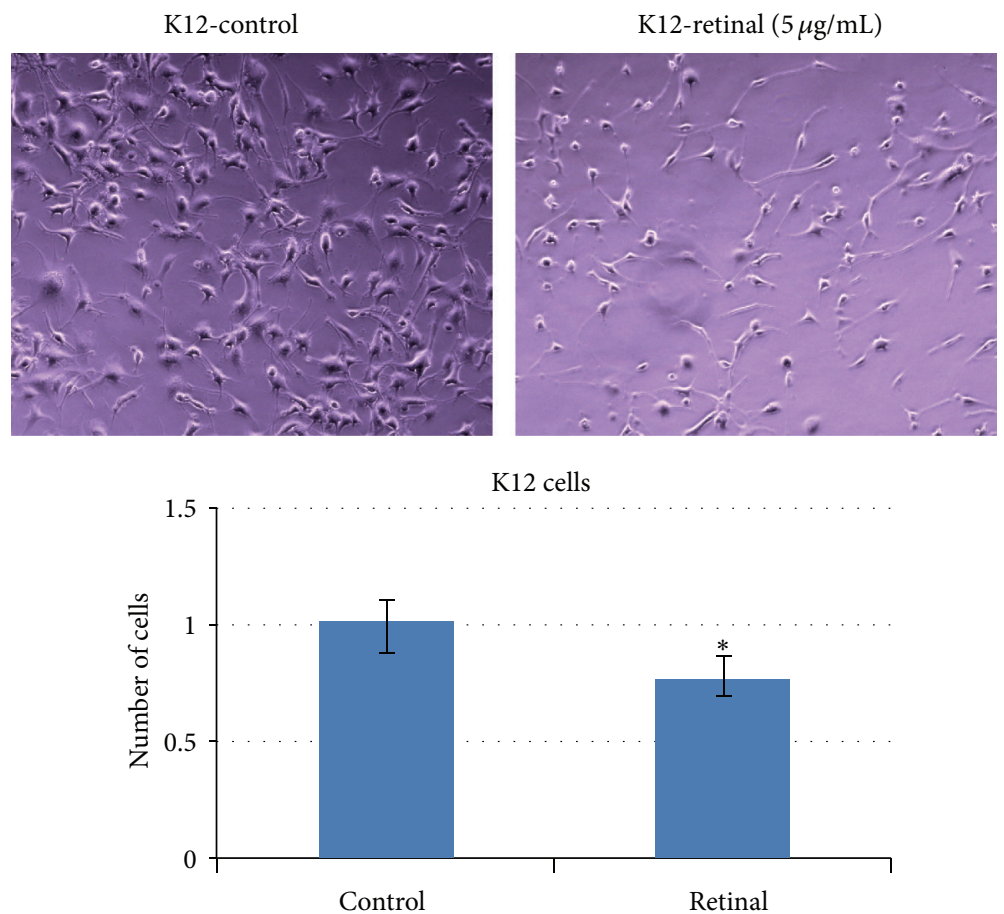

(b)

FIGURE 1: Retinal treatment of K7M2 cells decreased cell proliferation or survival. (a) and (b) The cell proliferation or survival capacity of K7M2 cells was more reduced with retinal treatment ( $5 \mu \mathrm{g} / \mathrm{mL}$, 2 days), compared to K12 cells. 


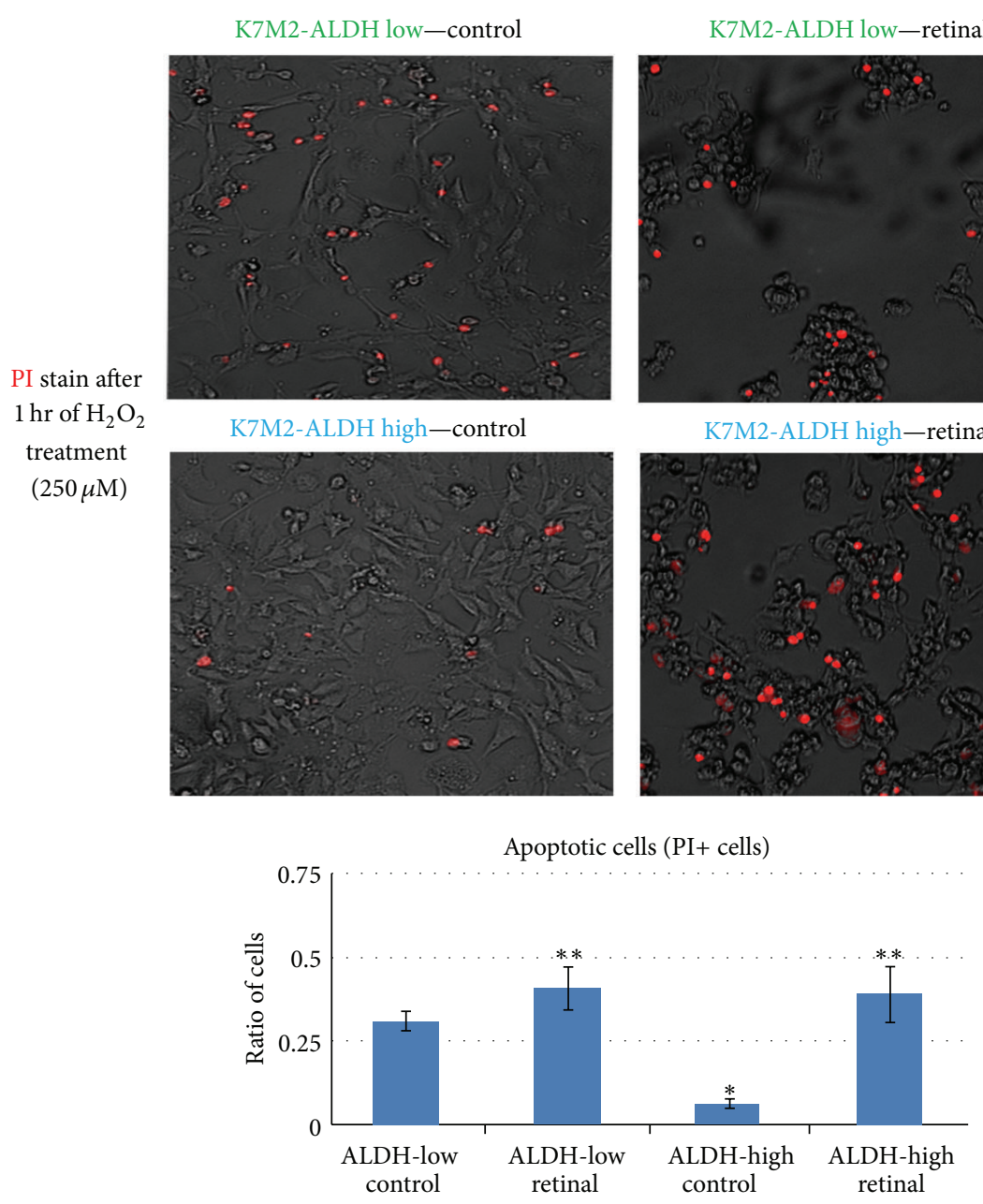

FIgURE 2: Retinal treatment of sorted ALDH-high K7M2 cells decreased antioxidative stress capacity. After cell sorting according to ALDH activity, it showed that the K7M2-ALDH high cells were more resistant to oxidative stress $\left(\mathrm{H}_{2} \mathrm{O}_{2}, 250 \mu \mathrm{M}\right.$ for $\left.1 \mathrm{hr}\right)$ than the K7M2-ALDH low cells, while the retinal treatment of K7M2-ALDH high cells was able to greatly decrease their resistance to oxidative stress and resulted in more cell apoptosis. “*” indicates that ALDH-high control cells are significant different $(p<0.05)$ compared to ALDH-low control cells; “**” indicates retinal treated ALDH-high or ALDH-low cells are significant different $(p<0.05)$ compared to nontreated ALDH-high or ALDH-low control cells, respectively.

Additionally, use of retinal as a therapeutic agent not only employs the antiproliferative properties of retinoic acid but also takes advantage of certain factors produced by tumor stem cells specifically. The action of retinal on the cells is mediated by retinoic acid [2]. Given that the conversion of retinal to retinoic acid requires the action of dehydrogenases, it can be expected that the activity of retinal will be higher in tumor stem cells, which produce much higher quantities of $\mathrm{ALDH}$, thereby making retinal therapy highly specific. Our results, therefore, indicate that retinal may be used as a cellular "Trojan Horse" of sorts to specifically target cancer stem cells.

Since pulmonary metastasis is the major cause of mortality in OS patients, it is crucial to devise therapeutic options that specifically target the metastatic potential of OS cells. Actin staining was performed to characterize the changes in cell morphology following treatment with retinal. We have previously demonstrated that changes in K7M2 cell shape and appearance of invadopodia relate to alteration in cells' migration/invasion potentials [17-21]. Our results demonstrate that in addition to acting as an antiproliferative agent, retinal also decreased cell invasiveness. Based on these results, we conclude that retinal therapy has the potential to specifically target metastatic OS cells and thus potentially decrease the incidence of pulmonary metastases, the ultimate cause of mortality in OS.

One of the limitations of this study is that the results are based on experiments carried out in vitro. While several studies have verified that the behavior of K7M2 cells parallels the growth of human OS cells, it is essential to conduct in vivo studies in order to validate these results.

\section{Conclusion}

Our current results demonstrate that retinal was capable of specifically acting on OS cells which express high levels of 


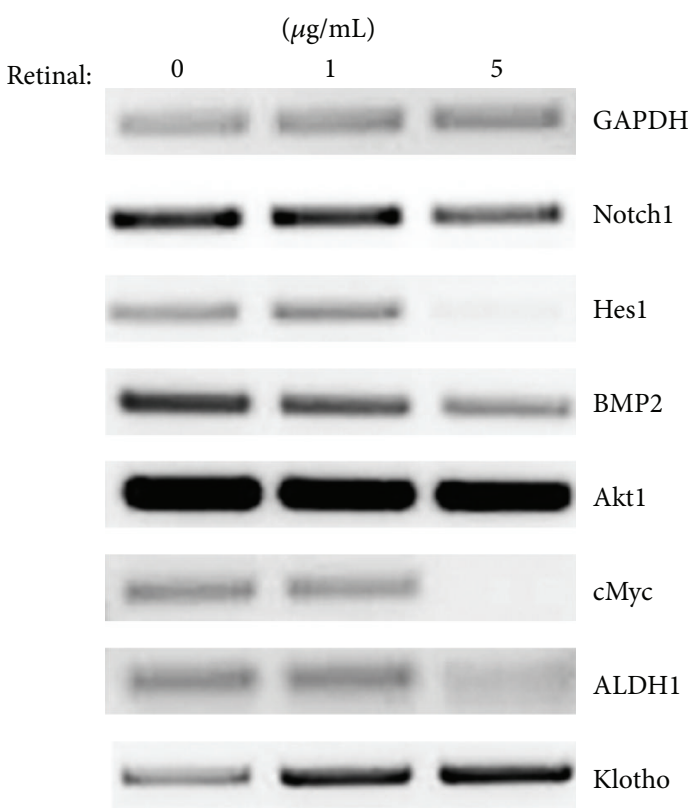

(a)
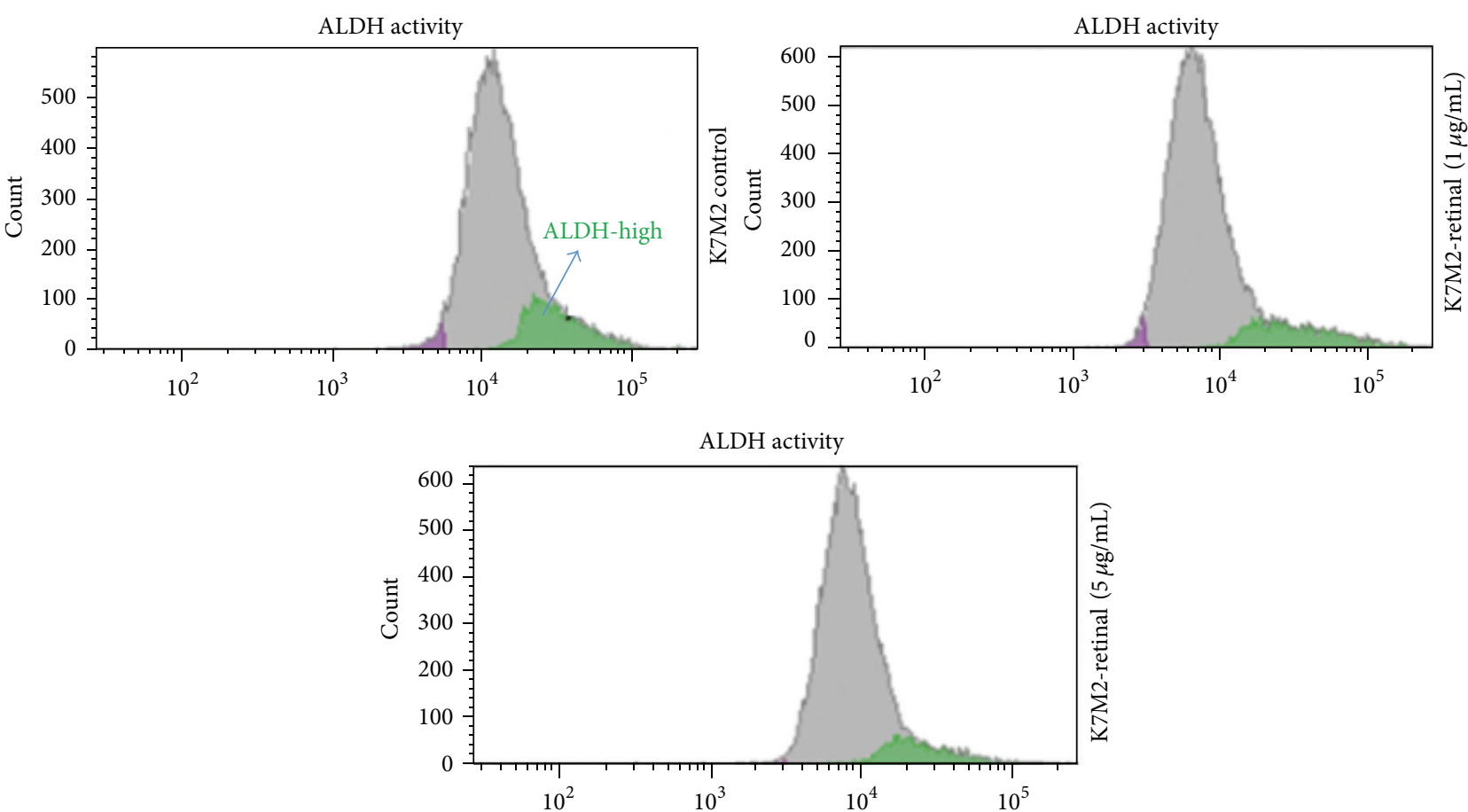

(b)

FIGURE 3: Retinal treatment of K7M2 cells modified the expression of metastasis-related genes and reduced ALDH activity. (a) RT-PCR results showed retinal treatment of K7M2 cells ( 1 or $5 \mu \mathrm{g} / \mathrm{mL}$ for 2 days) modified the expression of some key genes related with metastasis or tumorigenesis. (b) ALDH activity assay showed that retinal treatment of K7M2 cells reduced the aLDH activity of the cells.

ALDH and promoted these cells to undergo apoptosis. As cancer stem cells typically feature high ALDH activity, retinal could be used to specifically repress the growth of cancer stem cells with enhanced metastatic potential. Our study is the first to demonstrate the potential application of retinal for the treatment of tumorigenesis. We suggest that retinal could be potentially used to target OS cells with stem-like properties and ultimately decrease the ability of these cells to metastasize.

\section{Abbreviations}

ALDH: Aldehyde dehydrogenase retinal: All-trans-retinal 


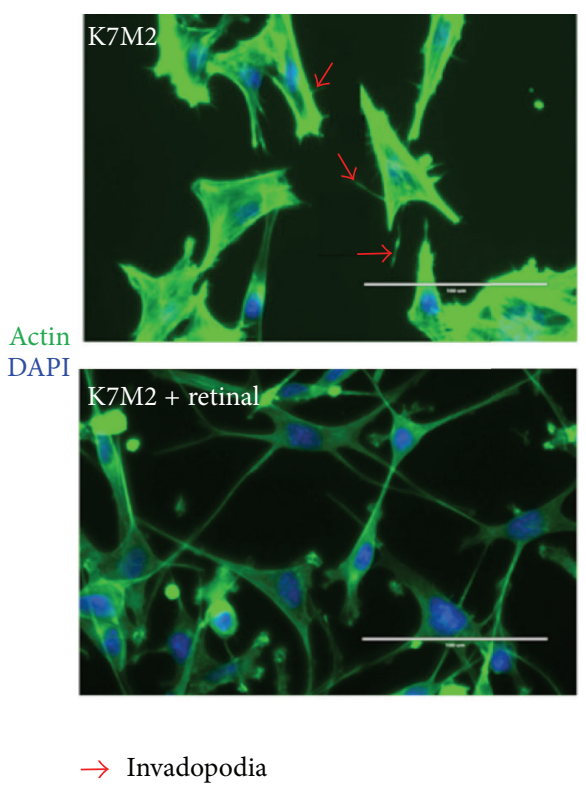

(a)

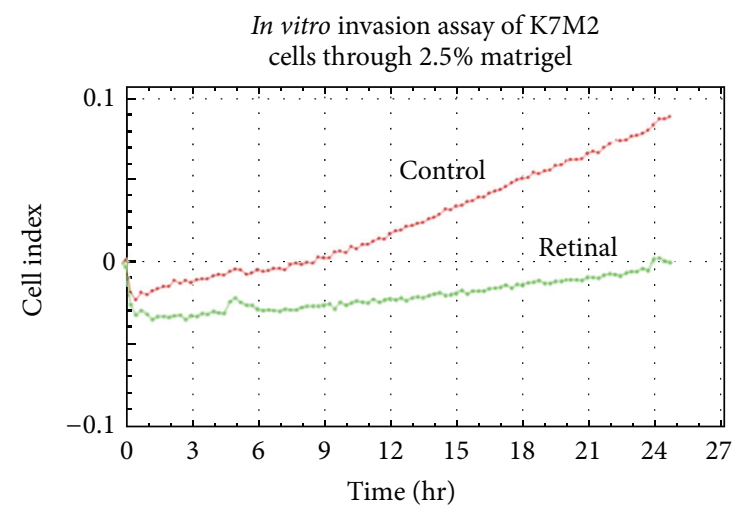

(b)

FIGURE 4: Retinal treatment of K7M2 cells changed migration-related cell morphological characteristics and decreased migration capacity. (a) Actin staining with phalloidin revealed that the cell morphology of K7M2 cells was modified with retinal treatment $(5 \mu \mathrm{g} / \mathrm{mL}$ for 2 days), showing reduced presence of invadopodia. (b) In vitro invasion assay with 3D-matrigel (2.5\%) revealed that K7M2 cells treated with retinal treatment $(5 \mu \mathrm{g} / \mathrm{mL}$ for 2 days) migrated slower than nontreated $\mathrm{K} 7 \mathrm{M} 2$ control cells.

FACS: Fluorescence-activated cell sorting

BMP: Bone morphogenetic protein

mTOR: Mammalian target of rapamycin.

\section{Conflict of Interests}

The authors declare that there is no conflict of interests regarding the publication of this paper.

\section{Acknowledgments}

The authors would acknowledge the funding support from NIH granted to Dr. Kurt Weiss (K08 CA177927), Sarcoma Foundation of America (SFA), University of Pittsburgh Cancer Institute, Pittsburgh Cure Sarcoma, and the Houy family in loving memory of Jon Houy.

\section{References}

[1] X.-H. Tang and L. J. Gudas, "Retinoids, retinoic acid receptors, and cancer," Annual Review of Pathology, vol. 6, pp. 345-364, 2011.

[2] N. Noy, "Between death and survival: retinoic acid in regulation of apoptosis," Annual Review of Nutrition, vol. 30, pp. 201-217, 2010.

[3] T. Schenk, S. Stengel, and A. Zelent, "Unlocking the potential of retinoic acid in anticancer therapy," British Journal of Cancer, vol. 111, pp. 2039-2045, 2014.

[4] M.-C. Chen, C.-Y. Huang, S.-L. Hsu et al., "Retinoic acid induces apoptosis of prostate cancer DU145 cells through Cdk5 overactivation," Evidence-Based Complementary and Alternative Medicine, vol. 2012, Article ID 580736, 11 pages, 2012.
[5] W. J. Huss, L. Lai, R. J. Barrios, K. K. Hirschi, and N. M. Greenberg, "Retinoic acid slows progression and promotes apoptosis of spontaneous prostate cancer," The Prostate, vol. 61, no. 2, pp. 142-152, 2004.

[6] C. Ginestier, J. Wicinski, N. Cervera et al., "Retinoid signaling regulates breast cancer stem cell differentiation," Cell Cycle, vol. 8, no. 20, pp. 3297-3302, 2009.

[7] M. E. Huang, Y. C. Ye, S. R. Chen et al., "Use of all-trans retinoic acid in the treatment of acute promyelocytic leukemia," Blood, vol. 72, no. 2, pp. 567-572, 1988.

[8] U. C. Dräger, E. Wagner, and P. McCaffery, "Aldehyde dehydrogenases in the generation of retinoic acid in the developing vertebrate: a central role of the eye," The Journal of Nutrition, vol. 128, supplement 2, pp. 463S-466S, 1998.

[9] D. Raha, T. R. Wilson, J. Peng et al., "The cancer stem cell marker aldehyde dehydrogenase is required to maintain a drug-tolerant tumor cell subpopulation," Cancer Research, vol. 74, no. 13, pp. 3579-3590, 2014.

[10] P. Marcato, C. A. Dean, C. A. Giacomantonio, and P. W. K. Lee, "Aldehyde dehydrogenase its role as a cancer stem cell marker comes down to the specific isoform," Cell Cycle, vol. 10, no. 9, pp. 1378-1384, 2011.

[11] E. Charafe-Jauffret, C. Ginestier, F. Iovino et al., "Aldehyde dehydrogenase 1-positive cancer stem cells mediate metastasis and poor clinical outcome in inflammatory breast cancer," Clinical Cancer Research, vol. 16, no. 1, pp. 45-55, 2010.

[12] S. Deng, X. Yang, H. Lassus et al., "Distinct expression levels and patterns of stem cell marker, aldehyde dehydrogenase isoform 1 (ALDH1), in human epithelial cancers," PLoS ONE, vol. 5, no. 4, Article ID e10277, 2010.

[13] K. Honoki, H. Fujii, A. Kubo et al., "Possible involvement of stem-like populations with elevated ALDH1 in sarcomas for 
chemotherapeutic drug resistance," Oncology Reports, vol. 24, no. 2, pp. 501-505, 2010.

[14] J. Feng, Q. Qi, A. Khanna et al., "Aldehyde dehydrogenase 1 is a tumor stem cell-associated marker in lung cancer," Molecular Cancer Research, vol. 7, no. 3, pp. 330-338, 2009.

[15] F. Zhou, Y.-D. Mu, J. Liang et al., "Aldehyde dehydrogenase 1: a specific cancer stem cell marker for human colorectal carcinoma," Molecular Medicine Reports, vol. 11, no. 5, pp. 38943899, 2015.

[16] H. Li, F. Ma, H. Wang et al., "Stem cell marker aldehyde dehydrogenase 1 (ALDH1)-expressing cells are enriched in triplenegative breast cancer," The International Journal of Biological Markers, vol. 28, no. 4, pp. e357-e364, 2013.

[17] X. Mu, C. Isaac, N. Greco, J. Huard, and K. Weiss, "Notch signaling is associated with ALDH activity and an aggressive metastatic phenotype in murine osteosarcoma cells," Frontiers in Oncology, vol. 3, article 143, 2013.

[18] X. Mu, C. Isaac, T. Schott, J. Huard, and K. Weiss, "Rapamycin inhibits ALDH activity, resistance to oxidative stress, and metastatic potential in murine osteosarcoma cells," Sarcoma, vol. 2013, Article ID 480713, 11 pages, 2013.

[19] X. Mu, D. Brynien, and K. R. Weiss, “The HDAC inhibitor vorinostat diminishes the in vitro metastatic behavior of osteosarcoma cells," BioMed Research International, vol. 2015, Article ID 290368, 6 pages, 2015.

[20] X. Mu, B. Sultankulov, R. Agarwal et al., "Chick embryo extract demethylates tumor suppressor genes in osteosarcoma cells," Clinical Orthopaedics and Related Research, vol. 472, no. 3, pp. 865-873, 2014.

[21] N. Greco, T. Schott, X. Mu et al., "ALDH activity correlates with metastatic potential in primary sarcomas of bone," Journal of Cancer Therapy, vol. 5, no. 4, pp. 331-338, 2014. 


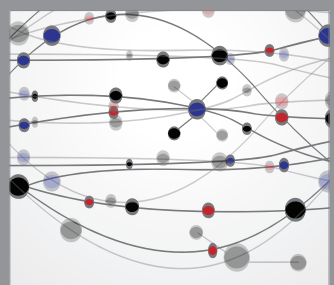

The Scientific World Journal
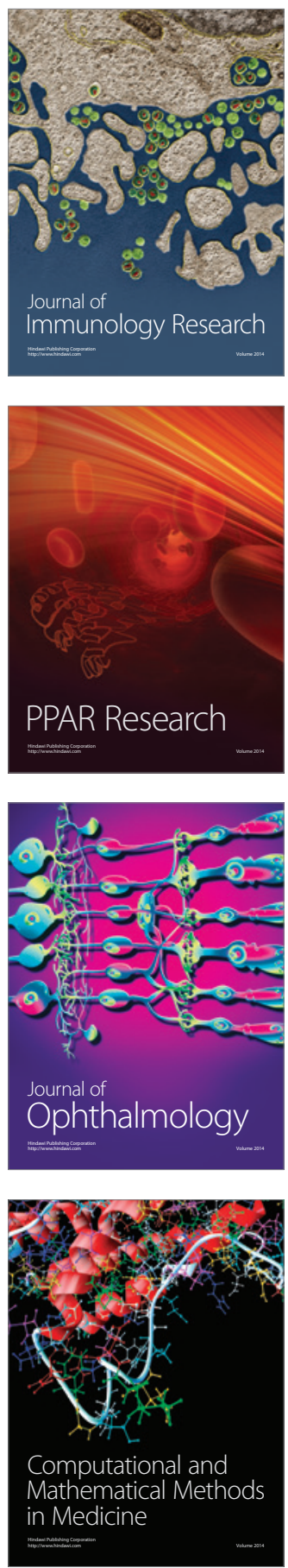

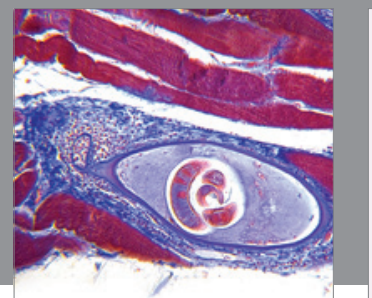

Gastroenterology

Research and Practice
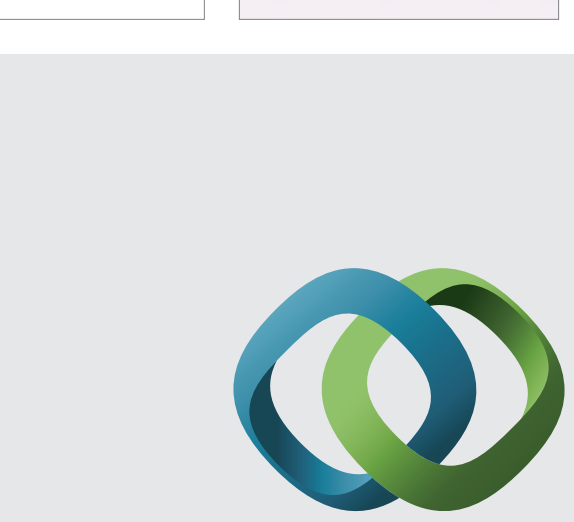

\section{Hindawi}

Submit your manuscripts at

http://www.hindawi.com
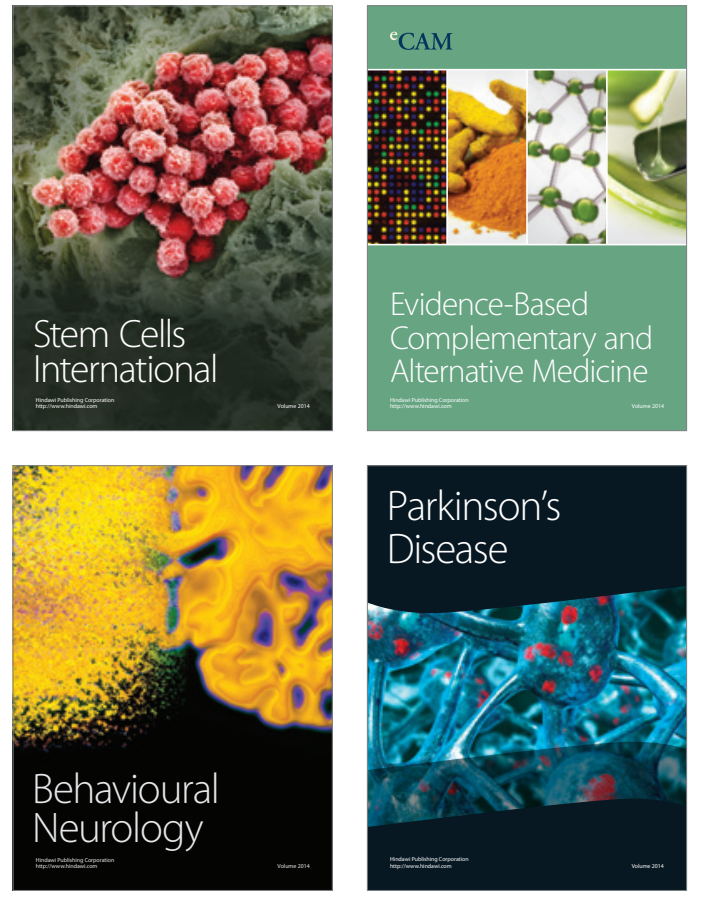
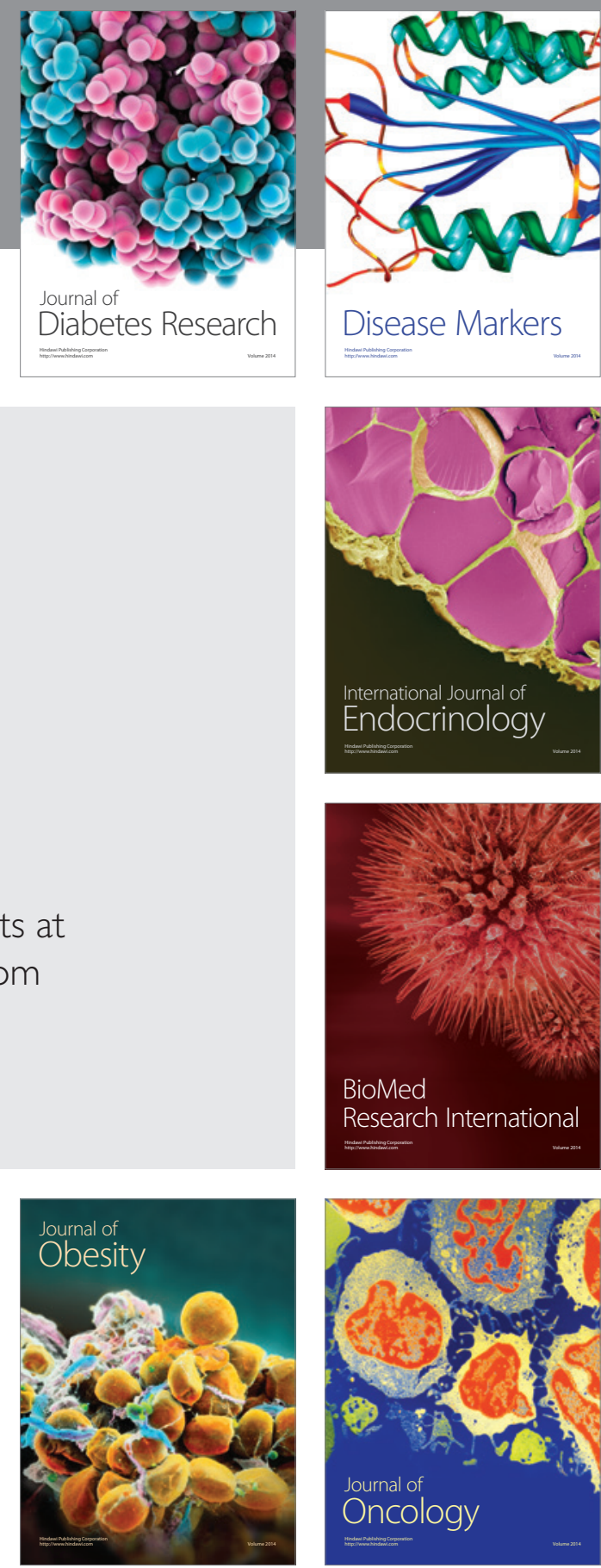

Disease Markers
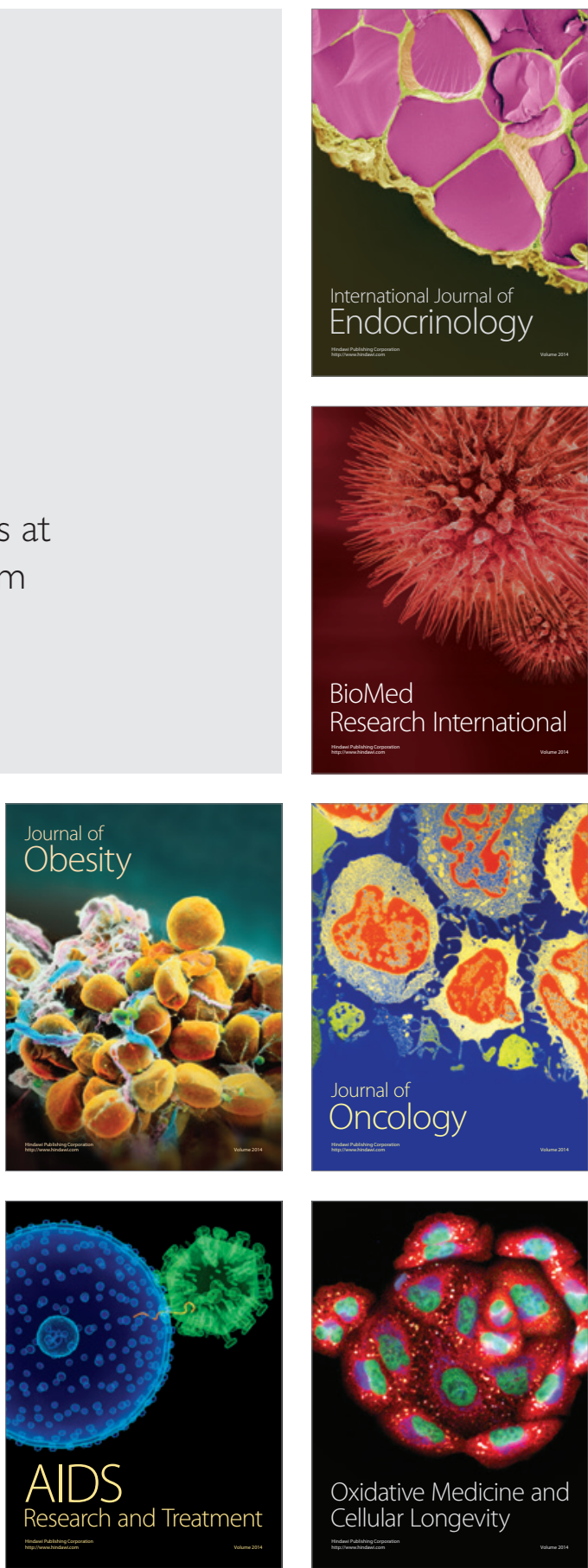